\title{
Congestion Management in Power System by Optimal Location And Sizing of UPFC
}

\author{
Dhanasekar.P ${ }^{1}$, Dr.K.Elango2 \\ ${ }^{l}$ Department of Electrical and Electronics Engineering, Valliammai Engineering College, Anna University, \\ India. \\ ${ }^{2}$ Professor, Department of Electrical and Electronics Engineering, Valliammai Engineering College, Anna \\ University, India.
}

\begin{abstract}
This paper presents a particle swarmoptimization (PSO) based algorithm to perform congestionmanagement by properplacement and sizing of one unified power flow controller (UPFC). The proposed approach makes use of the PSOalgorithm to allocate the near-optimal GenCos as well as the optimal location and size of UPFC whereas the Newton-Raphson solution minimizes the mismatch of the power flow equations. Simulation results (without/with the line flowconstraints, before and after compensation) are used to analyses the impact of UPFC on the congestion levels of the 5-bus test system.
\end{abstract}

\section{Introduction}

Promising idea has been rapidly developing over the last two decades for controlling the power flow in transmission lines with the application of flexible AC transmission systems (FACTS) through the utilization of large power converters. Different approaches have been presented for optimal placement of FACTS devices [1] including sensitivity analysis[2], congestion management by interline power flow controller and unified power flow controller (UPFC) [3].Application of artificial intelligent approaches for optimal congestion management is increasing in deregulated power systems. Recent approaches are mainly based on market models, particle swarm optimizations (PSOs) [4],genetic algorithms (GAs) and sensitivity analysis.Some studies have concentrated on maximizing social and individual welfare, as well as, social welfare considering reactive power and congestion management in deregulated environments. On the other hand, there have been a few studies on the UPFC application for congestion management. An artificial bee colony algorithm is proposed into minimize the generation fuel costs using UPFC unit[5].

This paper proposes a PSO-based algorithm for alleviatingcongestion in power systems by optimalplacement and sizing of one UPFC. The cost of UPFC is alsoincluded as the location index of merit in the optimization process. Simulations are performed to investigate the impactof UPFC oncongestion levels of the 5-bus test system.

\section{Mathematical Model Of Upfc}

In this paper, UPFC is selected to improve congestion management because of its flexibility and abilities in regulating the bus voltage and simultaneously controlling the active and reactive power flow.

\subsection{Power injection model of UPFC}

Newton-Raphson power flow formulation is used and UPFC is represented using the power injection model[6, 7], UPFC consists of two back-to-back voltage-source converters connected to power system through series and parallel power transformers.Impacts of UPFC on the network is reflected by a series connected voltage source $V_{T}$ and $W_{T}$, shunt current sources $I_{T}$ and $I_{q}$, connected to the network through series and shunt transformers as shown in Fig. 1. Therefore UPFC includes three adjustable parameters: voltage magnitude and phase angle of the series transformer $\left(V_{T}\right.$ and $\left.W_{T}\right)$ and reactive current $\left(I_{q}\right)$ of the shunt transformer. According to Fig. 1, UPFC can be modeled based on the followingequations

$$
\begin{gathered}
I_{i}=I_{T}+I_{q}+I_{i}^{\prime} \\
I_{T}=\frac{\operatorname{Re}\left[V_{T} \times I^{*}\right]}{V_{i}} \\
V_{i}^{\prime}=V_{T}+V_{i}
\end{gathered}
$$

The real and reactive power injections at buses $i$ and $j$ with a UPFC unit connected in lineij can be expressed as

$$
\begin{gathered}
S_{i j}=P_{i j}+j Q_{i j}=V_{i} \times I_{i j}^{*}=V_{i} \times\left(I_{i}+j V_{i} B / 2\right)^{*} \\
S_{j i}=P_{j i}+j Q_{j i}=V_{j} \times I_{j i}^{*}=V_{j} \times\left(j V_{j} B / 2-I_{i}^{\prime}\right)^{*}(5)
\end{gathered}
$$



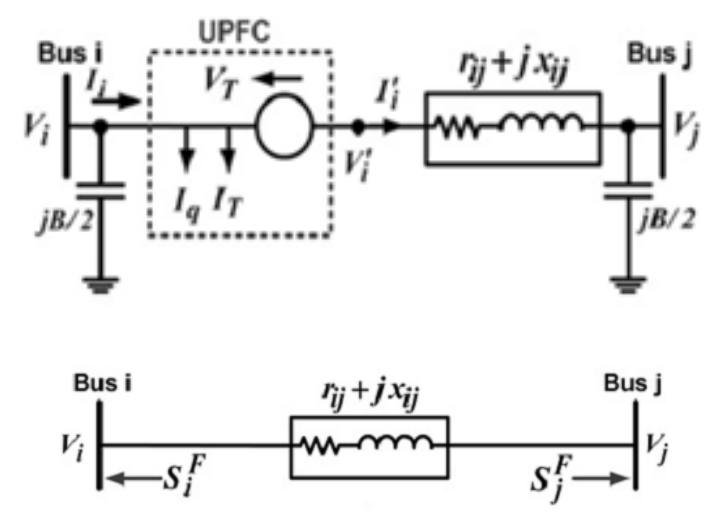

Figure.1 Model of transmission line with an UPFC

$$
\begin{array}{r}
P_{i s}=-g_{i j} V_{T}^{2}-2 V_{i} V_{T} g_{i j} \cos \left(\varphi_{T}-\delta_{i}\right)+V_{j} V_{T}\left[g_{i j} \cos \left(\varphi_{T}-\delta_{i}\right)+b_{i j} \sin \left(\varphi_{T}-\delta_{i}\right)\right](6) \\
P_{j s}=V_{j} V_{T}\left[g_{i j} \cos \left(\varphi_{T}-\delta_{i}\right)+b_{i j} \sin \left(\varphi_{T}-\delta_{i}\right)\right](7) \\
Q_{i s}=V_{i} V_{q}+V_{i} V_{T}\left[g_{i j} \sin \left(\varphi_{T}-\delta_{i}\right)+b_{i j} \cos \left(\varphi_{T}-\delta_{i}\right)\right] \\
Q_{j s}=-V_{j} V_{T}\left[g_{i j} \sin \left(\varphi_{T}-\delta_{i}\right)+b_{i j} \cos \left(\varphi_{T}-\delta_{i}\right)\right](9)
\end{array}
$$

Where $B, g_{i j}, b_{i j}, P_{i s}, Q_{i s}, P_{j s}$ and $Q_{j s}$ are line charging admittance, conductance of line ij, susceptance of line $\mathrm{ij}$, and active and reactive power injections at buses i and j, respectively. Equations (6)-(9) are added to the Jacobin matrix in load flow formulations.

\subsection{Cost of UPFC}

For more practical optimal placement and sizing of FACTS devices, it is recommended to also consider their investmentcosts in the $\mathrm{OF}[8]$.

$$
C_{U P F C}=0.0003 S_{U P F C}^{2}-0.2691_{U P F C}+188.22
$$

Where $C_{U P F C}$ and $S_{U P F C}$ are the total investment cost (in US\$/kVar) and the size (in MVar) of UPFC, respectively.

\subsection{Objective function}

\section{Problem Formulation}

In the market-based power systems, the conventional objective of market operator is to minimize the total generation cost. In this paper, the costs associated with congestion and voltage profile improvement are also included in the OF. Therefore we are faced with a more complex multi-objective optimization problem that includes load flow equality and operational inequality constraints

Where TGCi, and VVi are the total GenCos and voltage violation respectively.

$$
\min \left\{\sum_{i=1}^{n}\left(\frac{T G C_{i}}{T G C_{\text {Base }, i}}+\frac{V V_{i}}{V V_{\text {Base }, i}}\right)+n * C_{U P F C}^{\text {Annual }}\right\}
$$

\subsection{The GenCos cost functions}

To allocate the best network settings that minimize the overall generation cost function while imposing all network constraints. In this paper, the overall generation cost function is modeled by a quadratic function as follows

$$
\operatorname{TGC}\left(P_{G}\right)=\sum_{i=1}^{N_{G}}\left(a_{g i}+b_{g i} P_{G i}+c_{g i} P_{G i}^{2}\right)
$$

\subsection{The Voltage Violation}

Voltage violation is to allocate the best network settings that minimise the overall voltage violation while imposing all network constraints. In this paper, the overall voltage violation function is presented by the following quadratic function

$$
W=\sum_{i=1}^{N B} P F \times\left(V_{i}-1\right)^{2}
$$

Where $V_{i}$ is the voltage magnitude of bus $\mathrm{i}, \mathrm{NB}$ is the number of buses in the test system and PF is the voltage penalty factor.

\subsection{The UPFC cost function}

In this paper, one UPFC unit is used to minimise the total system cost (including the total GenCos and congestion costs) andimprove the voltage profile. 


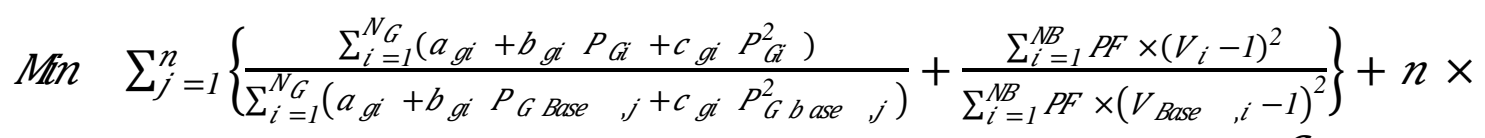

\subsection{Constraints}

In this paper, the $\mathrm{OF}(11)$ is subjected to the followingconstraints:

1. Power injection: The net injections of real and reactivepower at each bus are set to zero.

2. Generation limits: The limits on the maximum andminimum active (PG) and reactive $(\mathrm{QG})$ power generation ofthe generators are included as

$$
\begin{gathered}
P_{G}^{m i n} \leq P_{a} \leq P_{G i}^{m a x}, \quad Q_{G i}^{m i n} \leq Q_{G i} \leq Q_{G i}^{m x} \\
i=1,2, \ldots, N_{G}
\end{gathered}
$$

Where $P_{G i}$ and $Q_{G i}$ are the active and reactive powergeneration vectors at bus $G_{i}$, respectively.

Compensation limit: The maximum and minimum valuesof UPFC parameters are included as

$$
\begin{aligned}
& V_{T}^{m i n} \leq V_{T} \leq V_{T}^{m x} \\
& \varphi_{T}^{m i n} \leq \varphi_{T} \leq \varphi_{T}^{m x} \\
& I_{q}^{m i n} \leq I_{q} \leq I_{q}^{m x}
\end{aligned}
$$

\section{Development Of Proposed Pso}

One of the most difficult parts encountered in practical engineering design optimizations is handling constraints. Real-world limitationsfrequently introduce multiple, nonlinear and non-trivial constraints in the engineering design problems. Constraintsoftenlimitthefeasible solutionstoasmallsubsetofthedesignspace.A generalengineeringoptimizationproblemcanbedefinedasfollows:

\subsection{PSOBasedOPF}

Thoughawidevarietyofoptimizationtechniques singleobjectiveOPFproblemasmentionedearlierbuttheresults promisingandbetterascomparedtoothertechniques[13].Many ofPSOovertheothertechniquesinclude;-

Itisless susceptibleinbeingtrappedtolocalminima.

- Itcandealwithnon-differentiableobjectivefunctions.

- Itismoreflexibleand robust.

- Noproblemofprematureconvergence.

- Solutionqualityindependentoftheinitialpopulation.

\subsection{PSOAlgorithmforOPF problem}

Thevarious stepsinvolvedintheimplementationofPSOtotheOPFproblemare

Step 1:Inputparametersofsystem, andspecifythelowerandupperboundariesofeach variable.

Step2:Initializerandomlytheparticlesofthe population. These initialparticlesmustbe feasiblecandidatesolutions that satisfythepracticaloperationconstraints.

Step3:Toeachparticlesofthepopulation,employtheNewton-Raphsonmethodtocalculatepowerflow andthetransmissionloss.

Step 4:Calculatetheevaluation valueofeach particle, inthepopulationusing theevaluationfunction.

Step5:Compareeachparticle'sevaluationvaluewithitsgBest.Thebestevaluation valueamongthepBestis denotedas gBest.

Step6: Update thetimecountert $=\mathrm{t}+1$ 
Step7: Updatetheinertiaweightwgivenby

$$
W=W_{\text {max }}-\frac{W_{m a x}-W_{\text {min }}}{\text { iter }}=i \text { max }
$$

Step8:Modifythevelocityvofeachparticleaccording to the mentionedequation.

$$
V(k, j, i+1)=w * V(k, j, i)+C 1 * \text { rand } \quad *(p b e s t x \quad(j, k)-x(k, j, i)+C 2 * \text { rand } \quad *
$$

$$
\text { (gbestx } \quad(k)-x(k, j, i))(15)
$$

Step9:Modifythepositionofeachparticleaccordingtothementionedequation.Ifa particleviolates theits positionlimitsinanydimension, setits positionattheproperlimit

$$
x(k, j, i+1)=x(k, j-1, i)+v(k, j, i)(
$$

Step10:Eachparticleisevaluatedaccordingtoitsupdatedposition.Iftheevaluation particleisbetterthanthepreviouspBest, thecurrentvalueissettobe pBest.

valueofeach betterthangBest,thevalueis settobegbest.

IfthebestpBestis

Step11:i foneofthestoppingcriteriaissatisfiedthengotoStep12.Otherwise,goto Step6.

Step12:TheparticlethatgeneratesthelatestgBestis theoptimalvalue.

Theparametersthatmustbeselectedcarefullyfortheefficientperformanceof PSOalgorithmare:-

a. Both accelerationfactors $\mathrm{C}_{1} \& \mathrm{C}_{2}$.

b. Numberofparticles

c. Theinertiafactor.

d. The searchwillterminateifoneofthebellowscenariois encountered:

|gbest (i)-gbest (i-1) $\mid<0.0001$ for50iterations

Maximumnumberofiterationreached (500iterations)

e. Numberofintervals $\mathrm{N}$, whichdeterminethemaximumvelocityvk ${ }_{\mathrm{max}}$.

\section{Resultsand Discussion}

Matlabprogrammingcodesfor PSO and modified power flowalgorithm toincludeUPFCaredevelopedand incorporated together for the simulation purposes in this work.Thesuggestedalgorithmisappliedtothe5- bus test system.

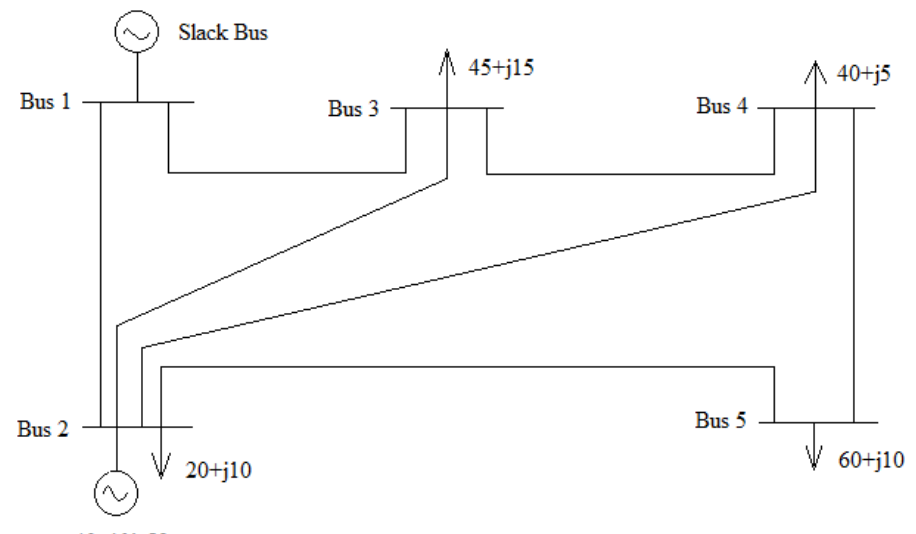

Figure 2.5 Bus test System 
Table -1 UPFC rating

\begin{tabular}{|c|c|}
\hline Parameter & Rating \\
\hline$X_{c r}$ & 0.1 \\
\hline$X_{v r}$ & 0.1 \\
\hline$P_{s p}$ & 0.4 \\
\hline$P_{\text {Sta }}$ & 1 \\
\hline$Q_{s p}$ & 0.02 \\
\hline$Q_{\text {Sta }}$ & 1 \\
\hline$V_{c r}$ & 0.04 \\
\hline$T_{c r}$ & $-87.13 / 57.3$ \\
\hline$V_{\text {crlo }}$ & 0.001 \\
\hline$V_{\text {crH }}$ & 0.2 \\
\hline$V_{u r}$ & 1.0 \\
\hline$T_{v r}$ & 0.0 \\
\hline$V_{\text {urLo }}$ & 0.9 \\
\hline$V_{\text {wht }}$ & 1.1 \\
\hline$V_{\text {irTar }}$ & 1.0 \\
\hline$V_{\text {ursta }}$ & 1.0 \\
\hline
\end{tabular}

Table-2 comparative result of Without UPFC and With UPFC

\begin{tabular}{|c|c|c|c|c|c|}
\hline \multicolumn{2}{|c|}{ Line } & \multicolumn{2}{c|}{ Without UPFC } & \multicolumn{2}{c|}{ With UPFC } \\
\hline From & To & $\begin{array}{c}\text { Line Flow } \\
\text { (MVA) }\end{array}$ & $\begin{array}{c}\text { Line Loss } \\
\text { (MVA) }\end{array}$ & $\begin{array}{c}\text { Line Flow } \\
\text { (MVA) }\end{array}$ & $\begin{array}{c}\text { Line Loss } \\
\text { (MVA) }\end{array}$ \\
\hline 1 & 2 & 90.251 & 9.210 & 92.334 & 9.025 \\
\hline 1 & 3 & 41.147 & 7.499 & 39.82 & 7.818 \\
\hline 2 & 1 & 87.636 & 9.210 & 89.643 & 9.025 \\
\hline 2 & 3 & 24.787 & 7.687 & 22.183 & 7.964 \\
\hline 2 & 4 & 28.098 & 7.416 & 31.161 & 7.032 \\
\hline 2 & 5 & 54.752 & 3.369 & 56.503 & 3.171 \\
\hline 3 & 1 & 39.629 & 7.498 & 38 & 7.818 \\
\hline 3 & 2 & 24.912 & 7.687 & 21.653 & 7.964 \\
\hline 3 & 4 & 20.488 & 4.160 & 18.521 & 4.193 \\
\hline 4 & 2 & 27.808 & 7.416 & 31.346 & 7.032 \\
\hline 4 & 3 & 19.245 & 4.160 & 15.955 & 4.193 \\
\hline 4 & 5 & 7.802 & 10.509 & 7.445 & 10.459 \\
\hline 5 & 2 & 53.8 & 3.369 & 55.539 & 3.171 \\
\hline 5 & 4 & 8.746 & 4.160 & 6.664 & 10.459 \\
\hline
\end{tabular}

\section{Conclusion}

Inthispaper,theeffectivenessoftheoptimallocationofUPFCforenhancingthesecurityofpowersystems by alleviate the congestionunder singleline contingencies has been investigated.A PSO technique has beensuccessfully appliedtotheproblem underconsideration. Alleviation of congestion is consideredas the optimization criterion.

\section{References}

[1] Hajforoosh. S.,Nabavi.S.M.H., and Masoum.M.A.S., "Coordinated aggregated-based particle swarm optimisation algorithm for congestion management in restructured power market by placement and sizing of unified power flow controller" IET Sci. Meas. Technol., Vol. 6, No. 4, pp. 267-278, 2012.

[2] Verma, K.S., Singh, S.N. and Gupta, H.O.: 'Location of unified power flow controller for congestion management', Electr. Power Syst. Res., Vol. 58, No. 2, pp. 89-96, 2001.

[3] Bruno, S., and LaScala, M.: 'Unified power flow controllers for security constrained transmission management', IEEE Trans. Power Syst., Vol. 19, No. 1, pp. 418-426, 2004

[4] Sarvi, M., Sedighizadeh, M., and Qarebaghi, J.: 'Optimal location and parameters setting of UPFC based on particle swarm optimization for increasing loadability', Int. Rev. Electr. Eng. (IREE), Vol. 5, No 5, Part B, pp. 2234-2240, 2010.

[5] Yousefi-Talouki, A., Gholamian, S., Hosseini, M., andValiollahi, S.:'Optimal power flow with unified power flow controller using artificial Bee colony algorithm', Int. Rev. Electr. Eng. (IREE), Vol. 5, No. 6, pp. 2773-2778, 2001.

[6] Jahani, R., ChahkandiNejad, H., ShafighiMalekshah, A., and Shayanfar,H.A.: 'A new advanced heuristic method for optimal placement of unified power flow controllers in electrical power systems', Int. Rev.Electr. Eng. (IREE), Vol. 5, No 6, pp. 2786$2794,2001$.

[7] LashkarAra, A., Aghaei, J., and Shabani, M.: 'Introducing a fuzzy inter active multi-objective approach to optimal location of UPFC', Int. Rev. Electr. Eng. (IREE), Vol. 5, No. 6, pp. 2896-2906, 2010

[8] Nor RulHasma Abdullah and Ismail Musirin and Muhammad Murtadha Othman.'Transmission Loss Minimization and UPFC Installation Cost using Evolutionary Computation for Improvement of Voltage Stability'International Middle East Power Systems Conference, 2010

[9] Singh, S.N., and David, A.K.: 'Optimal location of FACTS devices for congestion management', Elect. Power Syst. Res., Vol. 58, No. 2, pp. 71-79, 2001

[10] Mithulananthan, N.,andAcharya, N.: 'A proposal for investment recovery of FACTS devices in deregulated electricity markets', Electr. Power Syst. Res., Vol. 6, pp. 695-703, 2007.

[11] Verma, K.S., and Gupta, H.O.: 'Impact on real and reactive power pricing in open power market using unified power flow controller', IEEE Trans. Power Syst., Vol. 21, No. 1, pp. 365-371, 2006.

[12] Acharya, N., and Mithulananthan, N.: 'Locating series FACTS devices for congestion management in deregulated electricity markets', Electr. Power Syst. Res., Vol. 77, pp. 352-360, 2007

[13] Jizhong Zhu “OPTIMIZATION OF POWER SYSTEM OPERATION” A John Wiley \& Sons, Inc., Publication 